\title{
Lidil
}

Revue de linguistique et de didactique des langues

$58 \mid 2018$

L'enseignement et l'apprentissage de l'écrit académique à l'aide de corpus numériques

\section{Clara Romero, L'intensité et son expression en français} Paris, Éditions Ophrys, 2017, 280 p.

\section{Francis Grossmann}

\section{OpenEdition}

\section{Journals}

Édition électronique

URL : http://journals.openedition.org/lidil/5246

DOI : 10.4000/lidil.5246

ISSN : 1960-6052

Éditeur

UGA Éditions/Université Grenoble Alpes

Édition imprimée

ISBN : 978-2-37747-064-8

ISSN : $1146-6480$

\section{Référence électronique}

Francis Grossmann, «Clara Romero, L'intensité et son expression en français », Lidil [En ligne], 58 | 2018, mis en ligne le 02 novembre 2018, consulté le 25 septembre 2020. URL : http://

journals.openedition.org/lidil/5246 ; DOI : https://doi.org/10.4000/lidil.5246

Ce document a été généré automatiquement le 25 septembre 2020.

(c) Lidil 


\title{
Clara Romero, L'intensité et son expression en français
}

Paris, Éditions Ophrys, 2017, 280 p.

\author{
Francis Grossmann
}

\section{RÉFÉRENCE}

Clara Romero, L'intensité et son expression en français, Paris, Éditions Ophrys, 2017, 280 p.

1 L'ouvrage s'ouvre sur une introduction qui présente la notion d'intensité en linguistique, définie de manière assez extensive comme « la plus ou moins grande force associée à un message " (p.13). Cette approche large veut éviter de cantonner l'intensité aux "degrés d'intensité ", présentation traditionnellement effectuée dans les grammaires : dans l'approche classique, les adverbes quantifieurs (un peu, beaucoup, etc. ou le superlatif très) restent au centre de l'analyse, alors que dans celle qui est adoptée, l'intensité s'exprime à travers une grande diversité de moyens linguistiques, situés à différents niveaux (grammaticaux, lexicaux, discursifs, entre autres). La perspective proposée est donc originale, mais elle repose sur le pari risqué selon lequel, au-delà des différences de ses formes et de ses fonctions, il est possible de trouver une unité et une cohérence à la notion d'intensité, entendue dans ce sens très général. Le cadre d'analyse est éclectique, puisqu'on trouve des références, suivant les points traités, à la théorie de l'argumentation dans la langue de J.-C. Anscombre et O. Ducrot (pour les aspects argumentatifs), à la Théorie Sens-Texte de I. Mel'čuk et au LexiqueGrammaire de M. Gross (pour la partie lexicale), à la théorie des Actes de Langage et aux travaux des interactionnistes pour les derniers chapitres, etc. De nombreux travaux de linguistique française, portant sur des marqueurs précis, sont en outre convoqués, et chaque chapitre présente des pistes complémentaires de lecture.

2 L'ouvrage se veut pédagogique, avec des fiches, un glossaire, une table des illustrations, un très utile index des notions et formes, et un index des noms propres. Il fournit nombre de précisions indispensables sur le sujet, notamment à travers des 
éclaircissements terminologiques. Sa structure est claire, et se fonde sur les différents niveaux d'analyse linguistique ; ce parti pris a une contrepartie que l'on peut regretter : il évacue le continuum issu des phénomènes de grammaticalisation (malgré la place accordée au figement, en conclusion). Les deux premiers chapitres entérinent en effet la partition entre «expression grammaticale » et « expression lexicale » de l'intensité, tandis que le troisième chapitre, consacré aux structures phrastiques (l'expression grammaticale de l'intensité traitée dans le premier chapitre ayant été limitée aux mots et morphèmes grammaticaux) aurait pu mettre davantage en évidence l'articulation nécessaire entre les plans syntaxiques et discursifs. Leur disjonction aboutit en effet parfois à dissocier des analyses qui auraient mérité d'être regroupées : par exemple, la question des marqueurs discursifs, traitée dans le premier chapitre consacré à l'expression grammaticale, aurait gagné à être davantage connectée aux aspects interactionnels abordés en fin d'ouvrage. Peut-être est-ce là le prix à payer pour permettre une présentation commode, en forme de manuel.

Cependant, la question de fond est de savoir si lorsque nous parlons de l'intensité dans ce sens large, nous parlons toujours de la même chose. Ce problème n'est pas abordé de manière suffisamment frontale et précise. Sur le plan sémantique, la question traitée par Kleiber dans son article de 2013 (pourtant cité dans la bibliographie générale), qui distingue l'intensité comme "quantité qualitative » et l'intensité considérée en ellemême comme une propriété (par exemple dans le lexique des affects) n'est pas reprise et discutée. Un autre exemple concerne cette fois les frontières entre la dimension argumentative (dont l'analyse rappelle des concepts comme celui de force argumentative, mobilisés par les théoriciens de l'argumentation dans la langue) et l'intensité liée à la dimension énonciative (avec les questions de renforcement et d'atténuation). Ces deux aspects gagnent-ils à être conceptualisés à travers une même catégorie ? Peut-être que oui, mais un tel point de vue aurait mérité une argumentation plus approfondie. Le chapitre consacré aux structures phrastiques, qui examine le rôle des clivées et des pseudo clivées, ou encore celui consacré aux figures et tropes, qui intègre de manière pertinente la dimension rhétorique dans l'analyse linguistique fournissent des éléments de réponse intéressants, qu'il aurait été possible de mobiliser pour construire la démonstration. Peut-être aussi qu'une approche plus systématique, appuyée sur un large corpus textuel, aurait été utile, parce qu'elle aurait permis de mieux montrer, en contexte, l'intrication des différents procédés.

Ces quelques réserves ne doivent pas masquer les qualités d'ensemble et l'utilité de l'ouvrage, qui vient remplir un manque évident dans la bibliographie francophone, qui ne comportait aucune synthèse sur le sujet. En raison de sa richesse et de la finesse des analyses qu'il propose, il sera très utile aux étudiant.e.s. de sciences du langage ou de lettres, et à leurs enseignant.e.s ! 


\section{AUTEURS}

FRANCIS GROSSMANN

LIDILEM, Université Grenoble Alpes 